\title{
INCIDÊNCIA PÉLVICA: UM PARÂMETRO FUNDAMENTAL PARA DEFINIÇÃO DO EQUILÍBRIO SAGITAL DA COLUNA VERTEBRAL
}

\author{
PELVIC INCIDENCE: A FUNDAMENTAL PARAMETER FOR DEFINING THE SAGITTAL \\ BALANCE OF THE SPINE
}

INCIDÊNCIA PÉLVICA: UN PARÁMETRO FUNDAMENTAL PARA DEFINIR EL EQUILIBRIO SAGITAL DE LA COLUMNA

Sérgio Afonso Henneman ${ }^{1}$, Pedro Henrique Lacombe Antonell², Gustavo Carriço de Oliveira ${ }^{3}$

\section{RESUMO}

Este trabalho propõe um parâmetro anatômico, a incidência pélvica, como fator chave para a compreensão do alinhamento da coluna vertebral. Existe estreita relação, tanto para indivíduos normais como para indivíduos com deformidades da coluna, entre o parâmetro anatômico de incidência pélvica e a inclinação sacral, determinantes da lordose lombar. O ângulo de Cobb e a rotação vertebral apical servem de instrumento para o entendimento do aspecto tridimensional da coluna vertebral e das relações entre a pelve e a coluna. Uma equação preditiva para lordose é apresentada. A incidência pélvica parece ser o fator determinante do equilíbrio sagital da coluna. Ela determina as curvas da coluna vertebral, variando conforme outros parâmetros.

Descritores: Pelve; Lordose; Escoliose; Equilíbrio sagital; Postura

\begin{abstract}
This paper proposes an anatomical parameter, the pelvic incidence, as the key factor for managing the spinal balance. There is a close relationship for both normal individuals and those with spinal deformities between the anatomical parameter of pelvic incidence and the sacral slope, which determine lumbar lordosis. The Cobb angle and the apical vertebral rotation serve as a tool for understanding the three-dimensional aspect of the spine and its relationships with the pe/vis. A predictive equation of lordosis is postulated. The pelvic incidence seems to be the determining factor of the sagittal balance of the spine. It determines spinal curves and varies according to other parameters.
\end{abstract}

Keywords: Pélvis; Lordosis; Scoliosis; Sagital balance; Posture.

\section{RESUMEN}

En este trabajo se propone un parámetro anatómico, la incidencia de la pelvis, como el factor clave para entender la alineación de la columna vertebral. Hay estrecha relación, tanto para el normal y para las personas con deformidades de la columna entre el parámetro anatómico de la incidencia de la pelvis y la inclinación del sacro, determinantes de la lordosis lumbar. El ángulo de Cobb y la rotación vertebral apical sirven como una herramienta para entender el aspecto tridimensional de la columna vertebral y de su relación con la pelvis. Se presenta una ecuación de predicción para la lordosis. La incidencia pélvica parece ser el factor determinante del equilibrio sagital de la columna. Dicha ecuación determina las curvas de la columna y varía según otros parámetros.

Descriptores: Pelvis; Lordosis; Escoliosis; Equilibrio sagital; Postura.

\section{INTRODUÇÃO}

Apesar do alinhamento da coluna vertebral ter sido, durante muitos anos, avaliado apenas no plano coronal, diversos publicações destacam a importância que tem as alterações no plano sagital. Os autores também ressaltam a relação da coluna vertebral com as alterações na orientação pélvica e como elas determinam o alinhamento vertebral como um todo ${ }^{1-5}$. A morfologia espinhal sagital varia de um indivíduo para o outro e sua manutenção dentro de faixa de normalidade implica em menor gasto energético do indivíduo. Os músculos estáticos da região dorsal, responsáveis pela atividade anti-gravitária, podem sofrer sobrecarga na tentativa da manutenção indispensável do alinhamento espinhal. A compreensão da relação que a pelve tem com a coluna vertebral auxilia no entendimento e tratamento das alterações posturais de ambas regiões.

No entanto, o papel da anatomia pélvica neste posicionamento ainda não foi totalmente investigado. A incidência pélvica, parâmetro anatômico proposto neste estudo é fator determinante para a regulação do equilíbrio sagital. O alinhamento vertebral é concebido como o resultado de um posicionamento lordótico ideal da vértebra, acima de uma pelve corretamente orientada. Os valores do "Posicionamento lordótico ideal" e da "Pelve corretamente orientada" são definidos no presente estudo.

O presente estudo tem como objetivo apresentar os conceitos básicos de equilíbrio sagital, os diversos parâmetros determinantes do alinhamento vertebral e sua implicação no tratamento das deformidades vertebrais. Foi utilizado em grande parte, o estudo pioneiro de Legaye et al. ${ }^{6}$ que definiu os termos aqui apresentados.

1. Ortopedista, Chefe do Serviço de Coluna Vertebral do Hospital Mãe de Deus - Porto Alegre, RS, Brasil.

2. Ortopedista, Cirurgião de Coluna do Serviço de Coluna Vertebral do Hospital Mãe de Deus - Porto Alegre, RS, Brasil.

3. Mestre em Ortopedia eTraumatologia pela Faculdade de Ciências Médicas da Santa Casa de São Paulo, Cirurgião de Coluna do Hospital Regional de São José, São José, SC, Brasil. 


\section{Conceitos básicos de alinhamento da coluna vertebral}

1. Em relação às curvaturas da coluna vertebral, em pé, os parâmetros são os seguintes:

1.1 Ângulo da lordose lombar (L1-S1);

1.2 Ângulo da cifose torácica (T2-T12);

1.3 Linha de "prumo" (C7-S1).

2. Em relação à pelve, os parâmetros são: (Figura 1).

2.1 Incidência pélvica (IP), definida como o ângulo entre uma linha perpendicular ao ponto médio do platô sacral, e uma linha que liga este ponto até o eixo central da cabeça femoral. É considerado um parâmetro anatômico, constante, exclusivo para cada indivíduo, independente da orientação espacial da pelve, sendo a mobilidade da articulação sacro-ilíaca considerada insignificante e independente da idade uma vez que o crescimento ósseo esteja concluído. A IP pode ser determinada pela fórmula abaixo:

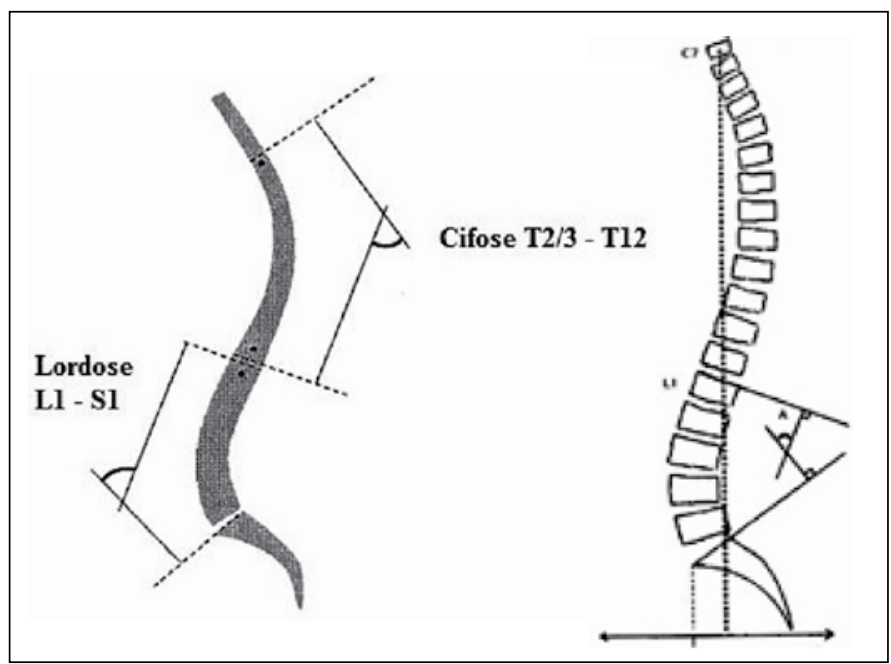

Figura 1. Os parâmetros 1.1, 1.2 e 1.3 são obtidos em radiografias em perfil.

Incidência pélvica (IP) = inclinação sacral (IS) + versão pélvica (VP)

2.2 Inclinação sacral (IS) é definida como o ângulo formado por uma linha entre o platô superior de S1 e uma linha horizontal (paralela ao solo). Quando a IS apresenta valor baixo diz-se que o sacro é vertical. Utiliza-se o termo sacro horizontal para um valor alto. Versão pélvica (VP), definida como o ângulo formado entre a linha que liga o ponto médio do platô sacral ao eixo da cabeça do fêmur e uma linha no eixo vertical (perpendicular ao solo).

Projeção de S1 (PS) em relação à cabeça femoral é definida como a distância entre o eixo bicoxofemoral e o ponto médio do platô superior sacral. É expressa em milímetros. Quando o ponto médio do platô sacral situa-se posteriormente ao eixo bicoxofemoral, a projeção de S1 é considerada positiva. Quando o ponto médio do platô situa-se anteriormente a esse eixo, a projeção de S1 é considerada negativa.

Estes últimos três parâmetros refletem a orientação sagital da pelve. Incidência pélvica (IP): o ângulo entre uma linha perpendicular ao ponto médio do platô sacral, e uma linha que liga este ponto até o eixo central da cabeça femoral.

Inclinação sacral (IS): o ângulo entre uma linha no platô superior de S1 e uma linha horizontal (paralela ao solo).

Versão pélvica (VP): o ângulo entre uma linha que liga o ponto médio do platô sacral até ao eixo da cabeça femoral, e uma linha vertical (perpendicular ao solo).

Projeção de S1 (PS): a distância entre o eixo bicoxofemoral e a projeção para este nível do ponto médio do platô superior sacral.

Estudo de Legaye et al. ${ }^{6}$ afirmam existir estreita relação entre a pelve e os parâmetros da coluna vertebral. Segundo os mesmos, a lordose lombar está intimamente relacionado com a orientação da pelve.

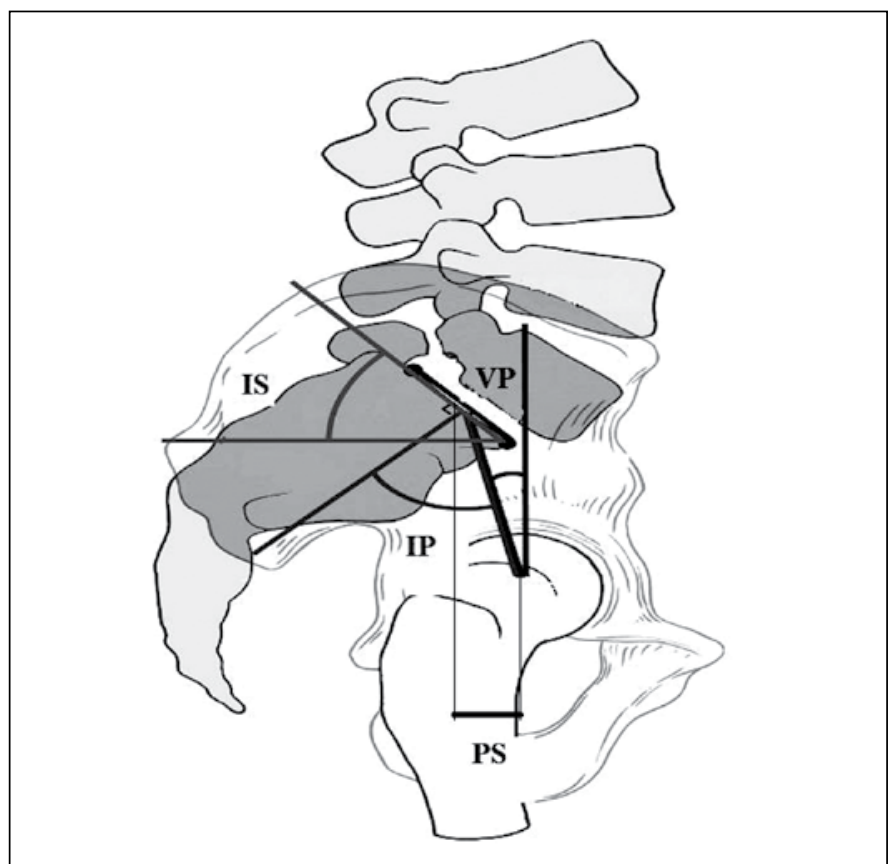

Figura 2. Parâmetros da pelve.

A orientação da pelve determina a posição sagital do platô superior sacral em relação à cabeça femoral, adaptada para cada indivíduo pelo parâmetro anatômico "incidência" Um valor baixo de incidência pélvica (IP) implica valores baixos de parâmetros pélvicos e uma lordose diminuída. Um valor elevado implica uma orientação pélvica bem inclinada e lordose acentuada. A incidência pélvica (IP) como citada anteriormente, é a soma algébrica da "inclinação sacral (IS)" e da "versão pélvica (VP)". Além disso, quanto maior o valor da incidência pélvica (IP), maior o valor da projeção de S1 (PS).

Para indivíduos normais, o parâmetro anatômico de incidência pélvica (IP) costuma ser uma constante para cada indivíduo. Ele determina os parâmetros da versão pélvica (VP), da projeção de S1(PS) e da inclinação sacral (IS).

Os valores normais na população adulta já foram definidos por vários autores ${ }^{7-11}$, (Tabela 01) sendo:

Nos pacientes com escoliose, existe uma adaptabilidade da pelve às condições espinhais sobrejacente, permitindo a manutenção de um equilíbrio sagital harmonioso.

Tabela 1. Valores normais da relação espino -pélvica

\begin{tabular}{c|c}
\hline Parâmetros & Graus $\left(^{\circ}\right)$ \\
\hline Incidência Pélvica (IP) & $40-65^{\circ}$ \\
\hline Versão Pélvica (VP) & $10-25^{\circ}$ \\
\hline Inclinação Sacral (IS) & $30-50^{\circ}$ \\
\hline Lordose Lombar & $40-80^{\circ}$ \\
\hline Cifose Torácica & $20-40^{\circ}$ \\
\hline
\end{tabular}

\section{DISCUSSÃO}

Existe estreita relação entre os parâmetros da pelve e as curvas da coluna vertebral. Qualquer alteração em um dos parâmetros como lordose, cifose torácica, versão pélvica, etc, induz uma mudança nos outros, exceto para a incidência pélvica (IP), que é o único parâmetro constante e anatômico, os outros sendo parâmetros posicionais. A incidência pélvica é um valor seguro para as variações inter-individuais da inclinação curvas lordóticas sacrais e lombares. Isto é verdade para a população normal e escoliótica. A influência da anatomia pélvica sobre a magnitude da curva sagital torna-se óbvia: uma grande incidência pélvica indica uma curva sacral acentuada e curvas sagitais de alto valor angular. 
A equação preditiva para a lordose lombar utilizada no planejamento do tratamento do desequilíbrio sagital, quando necessárias osteotomias de correção com base em parâmetros pélvicos, foi postulada Rose et al. ${ }^{12}$ como:

\section{$\mathrm{LL}=\mathrm{IP}+\mathrm{CT}-45$}

O ângulo de Cobb e de rotação apical, apesar de sua falta de correlação direta com lordose, afetam a relação entre os parâmetros pélvicos e os da lordose. Esta equação pode ser proposta para todos os indivíduos (normal e com escoliose).

A capacidade da unidade funcional coluna/ pelve para procurar manter um equilíbrio sagital da melhor forma possível, apesar da distorção frontal, depende tanto do parâmetro anatômico "incidência" e da adaptabilidade dos outros parâmetros.

O parâmetro de incidência pélvica (IP) tem o poder regulador tanto em indivíduos normais como naqueles escolióticos. Esta relação é tridimensional, levando em conta o parâmetro sagital espinal e pélvico, bem como parâmetros coronais. A presença de uma deformidade lateral da coluna, uma rotação vertebral de certa magnitude, perturba o relacionamento regular em termos de economia de energia.

A posição relativamente para frente do tronco aumenta o peso da carga sobre os níveis das vértebras lombares e no eixo bicoxofemoral ${ }^{13-16}$. O mesmo estudo de Legaye apresenta a primeira demonstração estatística do efeito da rotação axial vertebral na redução da lordose em pessoas com escoliose. Quanto maior os parâmetros para coluna vertebral (escoliose), mais restrita é a capacidade de compensação da curva lordótica quando a pelve altera os parâmetros posturais. Isso aumenta o risco de condições rentáveis de equilíbrio.

\section{CONSIDERAÇÕES FINAIS}

O parâmetro anatômico de incidência pélvica determina papel fundamental na regulação das curvas sagitais da coluna vertebral e na variabilidade individual da inclinação sacral e do grau de lordose. O objetivo deste sistema de regulação é garantir um equilíbrio econômico sagital através da manutenção das forças de peso suficiente atrás da coluna lombar e das cabeças coxofemorais, como tem sido demonstrado por estudos baricentrométricos ${ }^{14,15,17}$. É uma relação tridimensional entre a pelve e coluna vertebral.

Essa regulação sofre alterações quando ocorre uma deformidade da coluna. Quanto maior força rotação apical e o ângulo de Cobb da curva escoliótica, menor é a incidência pélvica, e mais restrita é a possibilidade de conseguir um equilíbrio sagital econômico. Esses resultados explicam por que a curva lordótica é freqüentemente reduzida em pacientes com escoliose.

Em suma, para os indivíduos submetidos a tratamento cirúrgico, atingindo valores normais de inclinação sacral e lordose lombar, provavelmente o gasto de energia será menor e oferecerá um melhor resultado funcional.

\section{REFERÊNCIAS}

1. Duval-Beaupère $G$, Robain $G$. Visualization on full spine radiographs of the anatomica connections of the centres of the segmental body mass supported by each vertebra and measured in vivo. Int Orthop. 1987;11(3):261-9.

2. Itoi E. Roentgenographic analysis of posture in spinal osteoporotics. Spine(Phila Pa 1976). 1991;16(7):750-6.

3. Graf $\mathrm{H}$, Hecquet J, Dubousset J. [3-dimensional approach to spinal deformities. Application to the study of the prognosis of pediatric scoliosis]. Rev Chir Orthop Reparatrice Appar Mot. 1983;69(5):407-16.

4. Legaye J, Duval-Beaupere G, Hecquet J, Marty C. Anatomical and barycentremetrical threedimensional conditions of adult lumbar and thoraco-lumbar adult scoliosis. Proceedings of the European Spinal Deformity, Society Meeting, Birmingham, 3 June; 1994.

5. Marnay T. L'équilibre du rachis et du bassin. Cahiers d'enseignemendte la SOFCOT. Paris: Elsevier; 1988. p. 281-313

6. Legaye J, Duval-Beaupère G, Hecquet J, Marty C. Pelvic incidence: a fundamental pelvic parameter for three-dimensional regulation of spinal sagittal curves. Eur Spine J. 1998;7(2):99-103.

7. Guigui P, Levassor N, Rillardon L, Wodecki P, Cardinne L. [Physiological value of pelvic and spinal parameters of sagital balance: analysis of 250 healthy volunteers]. Rev Chir Orthop Reparatrice Appar Mot. 2003;89(6):496-506

8. Vialle R, Levassor N, Rillardon L, Templier A, Skalli W, Guigui P. Radiographic analysis of the sagittal alignment and balance of the spine in asymptomatic subjects. J Bone Joint Surg Am. 2005;87(2):260-7.

9. Roussouly P, Gollogly S, Berthonnaud E, Dimnet J. Classification of the normal variation in the sagittal alignment of the human lumbar spine and pelvis in the standing position. Spine (Phila Pa 1976). 2005;30(3):346-53.
10. Barrey C, Jund J, Noseda O, Roussouly P. Sagittal balance of the pelvis-spine complex and lumbar degenerative diseases. A comparative study about 85 cases. Eur Spine J. 2007;16(9):1459-67.

11. Boulay C, Tardieu C, Hecquet J, Benaim C, Mouilleseaux B, Marty C, et al. Sagittal alignment of spine and pelvis regulated by pelvic incidence: standard values and prediction of lordosis. Eur Spine J. 2006;15(4):415-22.

12. Rose PS, Bridwell KH, Lenke LG, Cronen GA, Mulconrey DS, Buchowski JM, et al. Role of pelvic incidence, thoracic kyphosis, and patient factors on sagittal plane correction following pedicle subtraction osteotomy. Spine (Phila Pa 1976). 2009:34(8):785-91.

13. During J, Goudfrooij H, Keessen W, Beeker TW, Crowe A. Toward standards for posture. Postural characteristics of the lower back system in normal and pathologic conditions. Spine (Phila Pa 1976). 1985;10(1):83-7.

14. Duval-Beaupere G, Hecquet J, Dubousset J, Graf H, Tabuteau P, Roche P. Centre of mass supported by each vertebra on a 3D-image of the spine. In: 9th Annual Conference of the Engineering in medicine and Biology Society, Boston; 1987. p. 844-5.

15. Duval-Beaupere G. The sagittal shape of the spine and pelvis required for an economic standing position. In: Proceedings of the Congress of Societé Internationale de Recherche en Orthopedie et Traumatologie, Montréal, 1990.

16. Hecquet J, Legaye J, Santin JJ. Software "RACHIS 91". In: Proceedings of the International Symposium on 3-D Scoliotic Deformities. Montréal: Fisher Verlag, 1992. p. 26-33.

17. Duval-Beaupere G, Robain G. Les rapports anatomiques du point d'application de la masse du segment corporel supporté par chaque étage vertébral. Ann Kinesither. 1989;16(1):1-7. 